\title{
Botulinum toxin: Bio Warfare Agent and Vaccine Development
}

\author{
Arti Sharma* \\ Government Degree College Prithvipur, Niwari, Madhya Pradesh-472336, India
}

\begin{abstract}
Botulinum toxin is the bio warfare agent that is produced by obligate anaerobic bacteria called C. botulinum. It cause the botulism (Flaccid paralysis) by preventing the secretion of neurotransmitter (acetylcholine) from neurons. There are seven serotypes of botulinum toxin designated A-G, with limited therapies, and no USFDA approved vaccine for botulism. An investigational formalin-inactivated penta-serotype-BoNT/A-E toxoid vaccine was used to vaccinate people who are at high risk of contracting botulism. However, this formalin-inactivated penta-serotype-BoNT/A-E toxoid vaccine was losing potency and was discontinued. This chapter represents the different vaccines being developed to replace the discontinued toxoid vaccine. These vaccines include DNA-based and recombinant protein-based vaccines.
\end{abstract}

\section{Introduction}

Clostridium botulinum is a gram positive, an obligate anaerobic and endospore forming bacteria produce botulinum neurotoxins (BoNTs). It is the most potent neurotoxin so far known to humans ( $\sim 100$ billion times more toxic than cyanide) i.e. It is categorized as bio warfare category 'A' agent. It is divided into 8 serotypes designated $(\mathrm{A}-\mathrm{H})$ which have similar structure and differ antigenically. Few strains that secrete two types of toxins, i.e., Ba, $\mathrm{Ab}, \mathrm{Bf}$, and Af (capital letter denotes principal toxin type) [1]. Generally the serotypes A, B, E are associated with human clinical cases but rarely serotype $\mathrm{F}$, among the serotypes A and B are the most poisonous. It causes neuroparalytic disease in humans and animals called botulism [2]. BoNT is secreted as a single polypeptide chain which is cleaved endogenously or exogenously and converted into dichain [100 kDa heavy chain (HC) and $50 \mathrm{kDa}$ light chain (LC)] both chain allied by a disulphide bond. HC consist of binding and translocation domain at the carboxy (C) and amino $(\mathrm{N})$ terminal end respectively. HC-binding domain bind with the nerve membrane and its translocation domain facilitate the entry of light chain across the membrane into cytoplasm. Due to cytoplasmic $\mathrm{p}^{\mathrm{H}}$ variation the HC dissociates from LC. LC contain zinc endopeptidase catalytic activity that cleaves SNARE-complex (soluble $\mathrm{N}$-ethylmaleimide factor attachment protein receptor) which involved in docking and fusion of acetylcholine containing vesicles to post synaptic membrane which inhibit the liberation of the acetylcholine at the neuro-muscular junctions as a result autonomic nervous system will be paralyzed. This condition is called botulism or flaccid paralysis. Vaccination is the most effective medical countermeasure to prevent botulism. Characterization of new formalin-detoxified botulinum neurotoxin toxoids. Current commercial vaccines are toxoids (i.e., inactivated whole toxins). Toxoid vaccines have side effects such as local and systemic reactions that are increased in individuals who receive a second shot. To overcome the drawback of toxoid vaccines, researchers have studied a recombinant protein of the heavy chain to develop a vaccine. The most advanced recombinant-based vaccine is the recombinant botulinum toxin $\mathrm{A} / \mathrm{B}(\mathrm{rBV} \mathrm{A} / \mathrm{B})$ vaccine, which is currently undergoing clinical trials. However, no current Food and Drug Administration (FDA)-approved recombinant subunit vaccines exist for the prevention of botulism. Immunoproteomics is a science combining proteomics and immunology that facilitates screening many immunogenic proteins simultaneously. The spores are crucial to infection and persistence of $B$. anthracis, and the spores and vegetative cells can elicit a humoral immune response in the course of bacterial infections. Therefore we used the spore and the vegetative form of the B. anthracis vaccine strain A16R to identify immunogenic proteins that might augment the protective efficacy of PA-based vaccines. The most poisonous substance known to man, botulinum toxin has successfully established itself as a therapeutic agent over the years. Initially used to treat strabismus, botulinum toxin is now an accepted treatment in a wide spectrum of disorders and has over a 100 potential medical applications. The somewhat loosely-applied term wonder drug could not be better employed to describe the remedial potential of this agent. With time, and through further studies, it is likely that the number of conditions treated with botulinum toxin will keep expanding.

\section{Clostridium botulinum}

\section{Classification and characteristics}

C. botulinum is a Gram-positive; obligate anaerobic and endospore forming rod-shaped bacterium of 0.5-2.4 $\mu \mathrm{m}$ in width and 1.6-22.0 $\mu \mathrm{m}$ in length, with oval, sub terminal spores. The most unique feature of this bacterium is the production of botulinum neurotoxins (BoNTs), one of the most lethal naturally occurring toxins known to humans and animals. There are eight serotypes of this bacteria designated A to $\mathrm{H}$ are known on the basis of the different antigenic properties of their respective toxins (Figure 1) [3].

C. botulinum strains are divided into four different phenotypic groups (I-IV) based on biochemical properties. Group I strains are proteolytic types A, B and F with favorable growth temperature close to

*Correspondence to: Arti Sharma, Government Degree College Prithvipur, Niwari, Madhya Pradesh-472336, India, E-mail: arti.biochem05@gmail.com

Received: June 08, 2021; Accepted: June 25, 2021; Published: June 29, 2021 


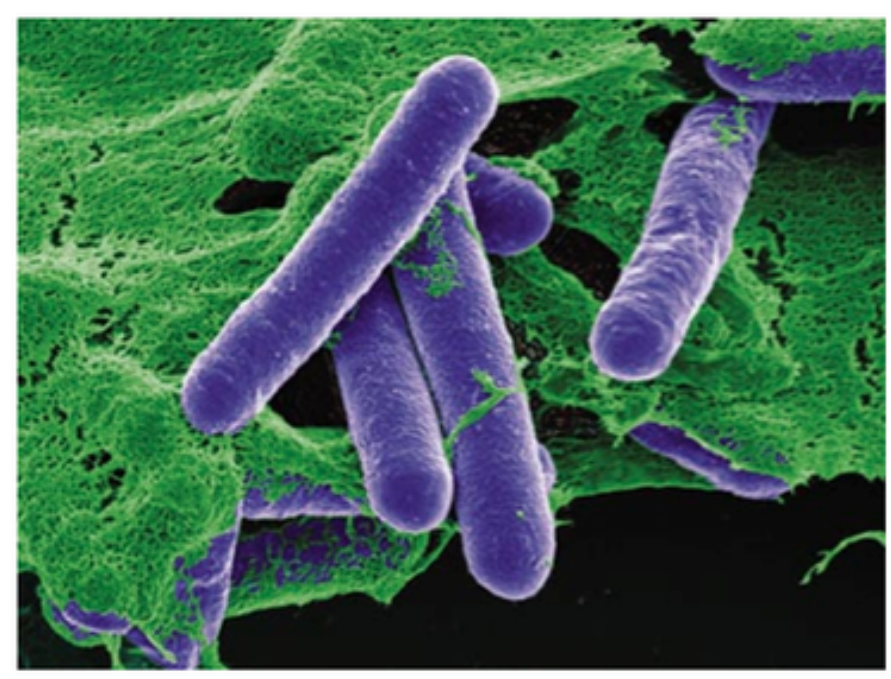

Figure 1. Electron micrograph of C. botulinum source

human body temperature. Their growth below $10^{\circ} \mathrm{C}$ is unlikely. Group II strains of types B, E and F do not produce proteolytic enzymes and are psychotropic. Group III organisms, types C and D have variable proteolytic activity, whereas group IV type G strains are proteolytic and vary from the other types by a lack of lipase production on egg yolk-containing agar plates. Type $\mathrm{G}$ has been also called Clostridium argentinense. Majority of human botulism cases belong to groups I and II strains even as group III strains are the most frequent causes of botulism in animals. Moreover to C. botulinum strains, Clostridium butyricum and Clostridium baratii are well-known to produce BoNT types $\mathrm{E}$ and F respectively [4].

\section{Historical background}

Botulinum neurotoxins are well famous in modern popular culture. However, its rise to fame began almost 200 years ago. In 1820s, the biological basis for food poisoning was not unstated. Dr. Justinus Kerner began to study a batch of improperly prepared blood sausages accountable for the death of several dozen Germans. Kerner stated that something was present in spoiled sausages that brought on the disease- something he called "wurstgift" (German for sausage poison). His experiments provided the better understanding of the neurological symptoms of food-borne botulism (ptosis, dysphagia, muscle weakness and if these symptoms left untreated results paralysis and respiratory failure. In 1895, a botulism outbreak after a funeral dinner with smoked ham in the small Belgian village of Ellezelles led to the discovery of the pathogen C. botulinum by Emile Pierre van Ermengem, Professor of bacteriology at the University of Ghent. In the early 1950s, Dr. Edward J. Schantz and his colleagues purified botulinum toxin type A into crystalline form. Physiologist Dr. Brooks discovered that injecting small amounts into a hyperactive muscle blocked the discharge of acetylcholine from motor nerve endings, causing temporary "relaxation" in 1953. In the 1960s, ophthalmologist Dr. Alan Scott started injecting BoNT/A into monkeys and stated that its muscle-relaxing effects might facilitate the treatment of strabismus. In 1978, Scott received FDA authorization to inject minute amounts of botulinum toxin into human volunteers and published numeral studies including a 1981 paper in the Transactions of the American Ophthalmological Society that asserted BoNTs "appears to be a safe, effective and useful therapy for strabismus".

Additional research explained the drug's benefits went beyond ophthalmology, given the patients with temporary relief from neck and shoulder spasms, facial spasms, even vocal cord spasms. Allergan introduced BOTOX $^{\circ}$ in 1989 , the first BoNT approved by the FDA to treat blepharospasm (eyelid spasms) and strabismus. In 2000, FDA permitted BOTOX therapy for cervical dystonia to reduce the brutality of abnormal head position and neck pain. On December 8, 2000, MyoBloc was accepted by the FDA for treatment of cervical dystonia, to diminish the severity of abnormal head position and neck pain. In 2002, FDA permitted BOTOX" for severe underarm sweating when topical medicines don't work well enough. In 2009, Dysport was approved by the FDA for the treatment of cervical dystonia to reduce the harshness of abnormal head position and neck pain. On August 2, 2010; the FDA announced the approval of Xeomin for the treatment of adults with cervical dystonia and for blepharospasm in adults [5].

\section{Prevalence}

C. botulinum is found in various environments including soil, decaying vegetation, streams, lakes and coastal waters. Surveys testing for the presence of $C$. botulinum in the environment were started in the US, Europe and Canada in the early 1920s. These surveys were carried out in response to numerous cases of food-borne botulism after the consumption of improperly prepared vegetables and meats. Much of this work was done before all of the types were identified and their characteristics are fully known; therefore, the data focus primarily on types A and B. The subsequent discovery of C. botulinum type $\mathrm{E}$ in the 1960s generated new investigations of its distribution. Data show that different types of $C$. botulinum are more prevalent in certain parts of the world, which has resulted in a close association between the types of botulism outbreaks and the occurrence of spores in the environment [6].

1. Types A and B are generally the cause of outbreaks in more temperate and warmer zones (with one type often predominating over the other).

2. Type A spores are found predominantly in soils of the Western US, Brazil, Argentina, China and the most frequently implicated foods are vegetables. They favor neutral to alkaline soils (average $\mathrm{pH}$ of 7.5) with low organic content, hence their virtual absence from the Eastern US and Europe, where the soil is heavily farmed.

3. Distribution of type B spores seems more uniform but those found in the soils of Eastern US are proteolytic and cases of foodborne illness usually involve vegetables. Those found in the UK and northern Europe is typically non-proteolytic and the vehicle is most often a meat product. Type B spores favor a slightly acidic soil $(\mathrm{pH}$ $6.25)$ and a higher organic content.

4. Type $\mathrm{E}$ is common in colder regions of the northern hemisphere and is mostly associated with fish and marine mammals. It is mostly prevalent in aquatic environments of the northern sub-arctic and temperate zones. High prevalence has been found in marine sediments in the coastal waters of Canada, Alaska, Greenland, Japan, Scandinavia and Russia. Freshwater sediments in various parts of the world such as US, Japan, Sweden and Finland have also been found to be contaminated with type E. Type E grows well in carrion of fish, marine mammals and invertebrates and these organisms also carry spores in their intestines. Water currents are significant in influencing the distribution of spores through the marine environment.

5. Types $\mathrm{C}$ and $\mathrm{D}$ mainly cause botulism in non-human species including cattle and birds. These types have been found in marshes inhabited by birds in localized areas around the world including the 
Gulf Coast and northwest in the US, in sediments in the Netherlands and Czechoslovakia, in Bangladesh, South Africa, Indonesia and Java and in specific regions of Japan, Thailand and New Zealand.

6. Type $\mathrm{F}$ is found in specific locations all over the world. In the US, it has been detected in sediment and soil from the Pacific Northwest, along with salmon from some rivers in Oregon and Washington. Isolates have been detected in soil from Switzerland, Paraguay and Argentina. It has also been found in certain locations in the UK, Taiwan, Japan, Brazil, the USSR, Indonesia and Java.

7. The first survey reporting a substantial number of C. botulinum type $\mathrm{G}$ was carried out in Switzerland. This type was first detected in soil in Argentina. Type $\mathrm{G}$ is difficult to detect in mixed cultures.

In all countries except Portugal, botulism is a statutory notifiable disease. The disease became notifiable at widely varying times, ranging from the end of the 1940s (England and Wales and Greece) to the start of the 1970s (Italy and Belgium) and very recently in Finland.

In India also the popularity of C. botulinum as well as botulism outbreaks were reported by several researchers. The first food borne botulism in India was reported in 1996 by Ramachaudhry, et al. in rural Gujarat resident school, out of 310 students 34 developed symptoms, two died and rest of them recovered back to normal after treatments which was mainly caused by C. butyricum [7]. In 2004, another food borne botulism case was reported in Ram Manohar hospital, New Delhi, in which the whole family developed symptoms of botulism within $48 \mathrm{hrs}$ after eating canned meat products [8]. Similar case was also reported from three year old boy admitted in KG hospital, Coimbatore. Prevalence of proteolytic C. botulinum type B from Indian tropical fish was reported by Lalitha \& Mahadeva lyer in 1990. Occurrence of $C$. botulinum in fresh and cured fish in retail trade in cochain were reported by Lalitha \& surendran in 2002 and the prevalence was around $19 \%(13 / 67)$ [9]. In fresh retail fish most of the samples were found positive for serotype A, B \& D. In pelagic fish $18 \%$ (7/39), whereas in demersal fish $21 \%(5 / 24)$ and in shrimp was $25 \%(1 / 4)$. Subsequently another study regarding genetic diversity among toxigenic clostridia was reported in 2009 [10]. The spores of type E were isolated from soil of Gwalior [11].

\section{Botulinum neurotoxin}

Botulinum neurotoxins are 150-kDa exo-toxins that are produced by Clostridium botulinum, C. butyricum and C. baratii. These neurotoxins are considered the most toxic substances on earth (the toxicity of BoNT is $\sim 100$ billion times more toxic than cyanide) and have been designated as category A biological threat agents. Botulinum neurotoxin has been developed as a biological weapon by many countries, including Japan, Germany, the United States, Russia and Iraq. The estimated human dose (assuming a weight of $70 \mathrm{~kg}$ ) of type A toxin lethal to $50 \%$ of an exposed population (the LD50) is estimated based on animal studies to be approximately 0.09 to $0.15 \mu \mathrm{g}$ by intravenous administration, 0.7 to $0.9 \mu \mathrm{g}$ by inhalation and $70 \mu \mathrm{g}$ by oral administration [12]. Botulinum toxins pose a major threat as biological weapons due to following reasons;

- They are extremely potent and lethal.

- Some of the toxins are relatively easy to produce and transport.

- People with botulism require prolonged intensive hospital care.

A deliberate release of botulinum toxin could be in the form of an aerosolized weapon or contamination of the food or water supply with C. botulinum or botulinum toxin. Several countries developed botulinum toxin as aerosol weapons in the past.

\section{Current threat of botulism neurotoxin}

Terrorists have previously attempted to use botulinum toxin as a bioweapon. Aerosols were discrete at numerous sites in city center Tokyo, Japan, and at US military installations in Japan on at least 3 occasions between 1990 and 1995 by the Japanese cult Aum Shinrikyo. These attacks were unsuccessful apparently because of defective microbiological technique, lacking aerosol-generating equipment or internal sabotage. The perpetrators obtained their $C$. botulinum spores in northern Japan soil. Development and employ of botulinum toxin as a probable bioweapon began at least 60 years ago. Japanese biological warfare group main head (Unit 731) gave to feeding cultures of C. botulinum to prisoners with fatal effect during that country's occupation of Manchuria which started in the 1930s. The US biological weapons agenda first used botulinum toxin during World War II. Germany had weaponized BoNTs, more than 1 million doses of botulinum toxoid vaccine were made for associated troops preparing to invade Normandy on D-Day. The US biological weapons plan was ended in 1969-1970 by administrative orders of Richard M. Nixon, then president. Research pertaining to biowarfare employ of botulinum toxin took place in other countries as well. Even though the 1972 Biological and Toxin Weapons gathering banned offensive research and manufacture of biological weapons, signatories Iraq and the Soviet Union consequently produced botulinum toxin for employ as a weapon. Botulinum toxin was 1 of numerous agents experienced at the Soviet site Aralsk-7 on Vozrozhdeniye Island in the Aral Sea. A Russian civil former senior scientist bioweapons plan reported that the Soviets had attempted splicing the botulinum toxin gene into other bacteria. With the financial difficulties in Russia after the termination of the Soviet Union, some of the thousands of researchers formerly engaged by its bioweapons agenda have been recruited by nations attempting to build up biological weapons. Iran, Iraq, North Korea, and Syria were listed by the US government as "state sponsors of terrorism" have developed or are whispered to be developing botulinum toxin as a weapon. After Persian Gulf War (1991), Iraq admitted to the United Nations examination team to having fashioned $19000 \mathrm{~L}$ of concentrated botulinum toxin of which about $10000 \mathrm{~L}$ were loaded into military weapons. These $19000 \mathrm{~L}$ of concentrated botulinum toxin are not entirely accounted for and comprise approximately 3 times the quantity desired to kill the whole existing human population by inhalation. In 1990 , Iraq deployed particularly designed missiles with a $600-\mathrm{km}$ range; 13 of these were packed with botulinum toxin, 10 with aflatoxin and 2 with Bacillus anthrax spores. Iraq also deployed special 400-lb (180-kg) bombs for instantaneous use; 100 bombs enclosed botulinum toxin, 50 enclosed anthrax spores and 7 contained maflatoxin. It is noteworthy that Iraq chose to weaponries additional botulinum toxin than any other of its known biological agents. Some fashionable analyses marked down the potential of botulinum toxin as a bioweapon because of constraints in concentrating and stabilizing the toxin for aerosol dissemination. However, these analyses concern to military uses of botulinum toxin to immobilize an antagonist. In contrast, deliberate discharge of botulinum toxin in a civilian population would be a source of substantial distraction and disruption. For example, it is predictable that a point-source aerosol liberate of botulinum toxin could kill $10 \%$ of persons within $0.5 \mathrm{~km}$ downwind. In addition, terrorist use of botulinum toxin might be manifested as deliberate infectious food. Mishandling of toxin in this manner could create either a large botulism outbreak from a single meal or episodic, broadly separated outbreaks. 31 in the United States, CDC maintained a well-established surveillance system 


\begin{tabular}{|c|c|c|c|}
\hline \multirow{3}{*}{ Type } & \multicolumn{3}{|c|}{ BoNT complex } \\
\hline & \multicolumn{2}{|c|}{ HA(+) complex } & HA $(-)$ complex \\
\hline & $16 \mathrm{~S}$ toxin & 19S toxin & $12 \mathrm{~S}$ toxin \\
\hline $\mathrm{A}(\mathrm{A} 1)$ & 11 & I & $\mathrm{m}$ \\
\hline B & $x=$ & & 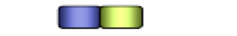 \\
\hline $\mathrm{C}$ & 口1 & & $\mathrm{C}$ \\
\hline $\mathrm{D}$ & $\mathrm{xl}$ & & $\mathrm{Co}$ \\
\hline $\mathrm{E}$ & & & 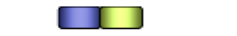 \\
\hline $\mathrm{F}$ & & & 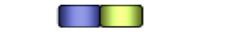 \\
\hline G & $\gamma$ & & \\
\hline
\end{tabular}

$\square$ BNT
NTNH

Figure 2. Characteristics of types A to G BoNT complexes. Type A (A1) BoNT is secreted by $C$. botulinum in three forms: $12 \mathrm{~S}, 16 \mathrm{~S}$ and $19 \mathrm{~S}$ toxins. Types B, C and D BoNT are secreted in two forms: $16 \mathrm{~S}$ and $12 \mathrm{~S}$ toxins. Types E, $\mathrm{F}$ (and A2) BoNT are secreted as $12 \mathrm{~S}$ toxin. Type G BoNT complex is secreted as $16 \mathrm{~S}$ toxin

for human botulism based on clinician reporting that would punctually detect such events $[13,14]$.

\section{Structural Aspects of Botulinum Neurotoxin Complex}

Overview of Molecular Composition of BoNT Complex: Botulinum neurotoxin (BoNT/NTX/8S toxin) is divided into eight serotypes, BoNT/A through BoNT/H, on the basis of their immunological properties. Moreover, the variation observed in BoNT protein sequences within the serotypes, in serotype A-F, has resulted in construction of BoNT subtypes within a serotype. For instance, five subtypes of BoNT/A have been known. Subtypes are differing by at least $2.6 \%$ at the amino acid level. These subtypes BoNT are secreted by the bacterium as complexes (progenitor toxins/BoNT complexes) allied with nontoxic components (nontoxic neurotoxin-associated proteins, NAP). Three forms of BoNT complexes, 12S toxin (M toxin/ M-TC), 16S toxin (L toxin/L-TC), and 19S toxin (LL toxin/LL-TC), are the main forms in cultures of the bacteria. $12 \mathrm{~S}$ toxin is contained of a BoNT and a nontoxic non hemagglutinin (non-toxic non-HA, NTNH, also called as NTNHA; $130 \mathrm{kDa}$ ). $16 \mathrm{~S}$ toxin is contained of a BoNT, an NTNH, and numerous hemagglutinin (HA) proteins. 19S toxin has the identical components as $16 \mathrm{~S}$ toxin and is supposed to be a dimer of two $16 \mathrm{~S}$ toxins allied by one of the HA proteins. C. botulinum type A (A1) strain released 12S, 16 S and $19 \mathrm{~S}$ toxins. Type B, C and D strains secreted $16 \mathrm{~S}$ and $12 \mathrm{~S}$ toxins. Type A2, E and F strains only secrete $12 \mathrm{~S}$ toxin. Type $\mathrm{G}$ strain secretes only $16 \mathrm{~S}$ toxin. Toxin types A, B, E and $\mathrm{F}$ cause both humans and animals botulism, whereas types $\mathrm{C}$ and $\mathrm{D}$ cause mainly animal botulism but very hardly in humans. Type G toxin secreting organisms have been isolated from soil but no naturally happening outbreaks of botulism caused by type $\mathrm{G}$ toxin have been reported (Figure 2) [15].

Subunit structure of botulinum toxin complexes: Botulinum toxin is encoded by two clusters (cluster-1, cluster 2). Cluster-1 encode $12 \mathrm{~S}$ complex and cluster- 1 and cluster- 2 both encode $16 \mathrm{~S}$ complex. $12 \mathrm{~S}$ complex contain NTNHA and BoNT and $16 \mathrm{~S}$ complexes contain heamoglutinin proteins (HA-1, HA-2 and HA-3). A medium toxin complex contains botulinum toxin and NTNHA $(300 \mathrm{kDa})$ and a large toxin complex that also includes the three HA molecules (500-600 $\mathrm{kDa})$. In contrast, serotypes $\mathrm{E}$ and $\mathrm{F}$ produce only the medium toxin complex. Serotype A forms a third complex with a higher molecular weight $(900 \mathrm{kDa})$. The whole molecular structure of BoNT type D large toxin complex has been visualized and contains a 14-subunit complex of neurotoxin, NTNHA, 3- HA3 molecules HA-70, three HA2 / HA-17, and 6-HA1/ HA-33). A denaturing capillary electrophoresis technique was performed to determine the subunits forming the higher molecular weight toxin complex of botulinum toxin type A, concluding that it comprises one-copy of the $150 \mathrm{kDa}$ BoNT and NTNHA subunits, as well as 5-6 HA-17, 4-5 HA-23, 3-4 HA-48, and 8-9 HA-34 subunits, with a total mass of $880-1000 \mathrm{kDa}$ (Figure 3 and 4) [16].

Functions of Complexing Proteins: NTNHA and HA proteins are the complexing proteins of botulinum neurotoxins. These proteins having following functions:

1. Complexing proteins defend the native neurotoxin from devastation in the gastrointestinal tract with oral ingestion.

2. These proteins have a job in the uptake and transcytosis of botulinum toxin through the intestinal epithelium to reach and affect muscle.

3. Complexing proteins limit botulinum toxin diffusion.

4. Complexing proteins are required to stabilize the neurotoxin (Figure 5).

Structure and Activity of BoNT: BoNT is a single-polypeptide chain of $150 \mathrm{kDa}$ but must be post-translationally modified by a bacterial or tissue protease for activation. The active form of the toxin consists of a light chain (LC, $50 \mathrm{kDa}$ ) and a heavy chain (HC, $100 \mathrm{kDa})$. Light chain contains catalytic domain and heavy chain contain translocation

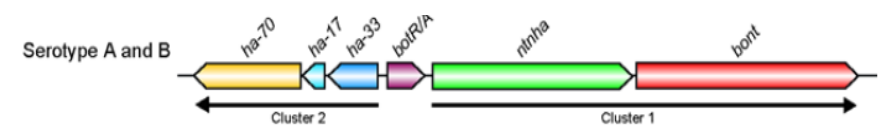

Figure 3. Depicts the genetic organization of botulinum toxin complex

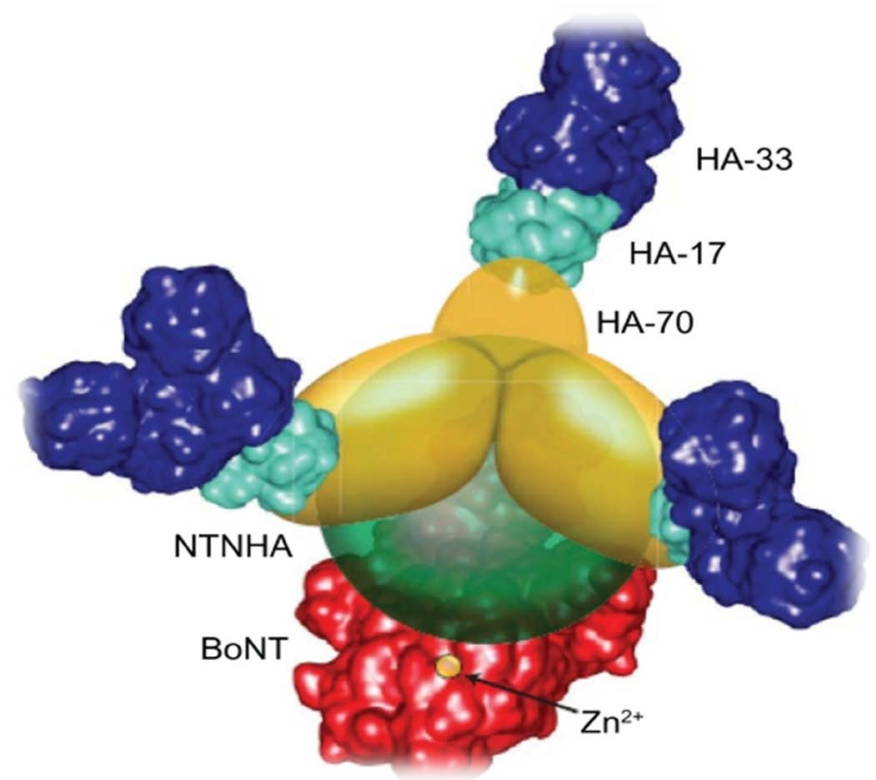

Figure 4. Depicts the arrangement of components in botulinum toxin type D complex. Botulinum toxin is highlighted in red, the nontoxic, nonhemagglutinin protein in green, three HA-70 in yellow, six HA-33 in blue, and three HA-17 in cyan. The catalytic zinc ion in botulinum toxin is indicated by the orange circle and the arrow. A novel subunit structure of $C$. botulinum serotype $\mathrm{D}$ toxin complex with three extended arms 


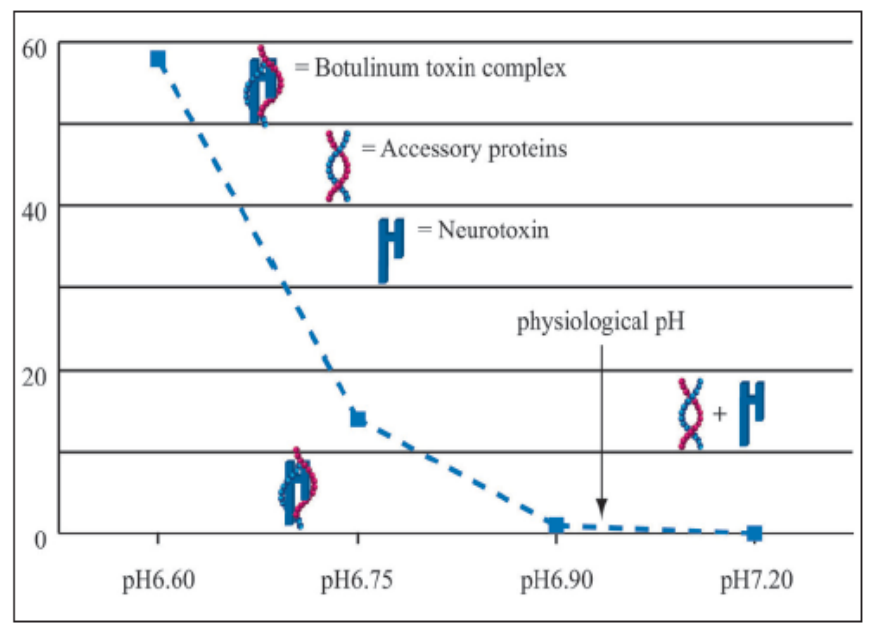

Figure 5. Dissociation of the botulinum toxin complex under physiological conditions. At physiological $\mathrm{P}^{\mathrm{H}}$, complexing proteins or accessory proteins (NTNHA and HA) are dissociated from the botulinum neurotoxin and it is going to be free for its biological function [28]

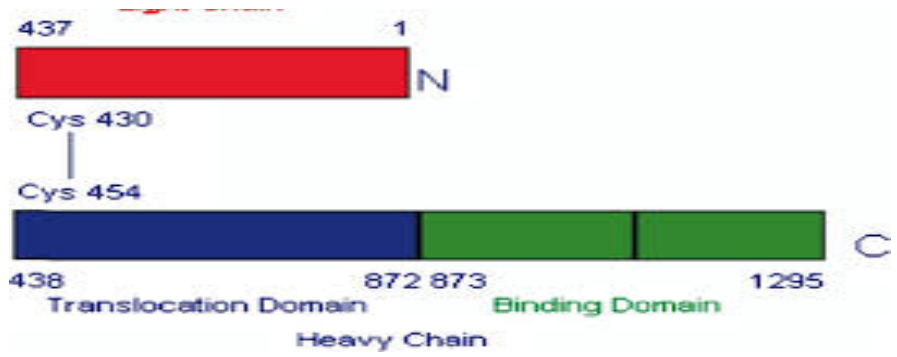

Figure 6. Depicts the di-chain structure of a BoNT/A. Botulinum neurotoxins are $\sim 150-k D a$ single-chain polypeptides and post-translationally nicked to form di-chain molecules. The light and heavy chains of BoNT/A are allied by a single disulphide bond, Cys430-Cys454. The light chain is depicted in red, functions as zinc-dependent endopeptidase. The heavy chain contains two functional domains of approximately equal size. The $\mathrm{N}$-terminal part is shown in blue, is the translocation domain and is thought to be implicated in translocation and activation of the $\mathrm{LC}$. The $\mathrm{C}$-terminal part is shown in green, is acting as binding domain

and binding domain at the end of $\mathrm{C}$ and $\mathrm{N}$ terminus respectively. The chains remain covalently and reversibly coupled by a disulphide bond until exposed to reducing conditions, such as in the nerve cytosol (Figure 6) [17].

Mode of action of BoNTs: The botulinum toxin's mechanism involves three steps:

1. Extracellular binding and internalization

2. Membrane translocation and intracellular substrate cleavage

3. Blockage of Ach release

\section{Extracellular binding and internalization}

Botulinum neurotoxin goes and binds with GT1b receptor (glycoprotein) which is present upon the surface of postsynaptic neuronal membrane through the C-terminus domain of the heavy chain. This specific docking is the reason for BoNTs high selectivity for cholinergic synapses.

2. Membrane translocation and intracellular substrate cleavage

After binding, it is reached into cytoplasm by $\mathrm{N}$-terminus domain of heavy chain. Due to cytoplasmic $\mathrm{pH}$ variations heavy chain dissociate from light chain. Light chain that contain zinc-endo-peptidase activity, cleaves respected SNARE proteins (light chain BoNTA/C/E cleave SNAP-25, BoNT/C also cleaves syntaxin and BoNT/B/D/F/G cleave VAMP-2).

\section{Blockage of Ach release}

Proteolytic cleavage of the SNARE protein complex by light chain prevents the docking of the acetylcholine vesicles on the inner surface of the pre synaptic membrane as a result exocytosis of acetylcholines containing vesicles is blocked that causes muscle paralysis as a result autonomic nervous system (ANS) dysfunction and this condition is called flaccid paralysis or botulism. Even though the toxin acts mainly on cholinergic nerve endings, it does have the capability to stop exocytosis from other nerve endings as well. By disrupting neurotransmission at cholinergic junctions in the ANS, the toxin can also affects various forms of autonomic dysfunction. Its most life-threatening potential however is its capability to discontinue respiration by disrupting neurotransmission in diaphragm and intercostal-muscles (Figure 7).

\section{Botulism}

Botulism is a neuroparalytic condition caused by botulinum neurotoxins. The neurotoxin can go through into body via the gastrointestinal tract/mucous membranes/the respiratory tract. After entering the body, the botulinum neurotoxins are absorbed into the blood and lymphatic circulation which then arbitrate the toxin to motor nerve endings where it blocks the acetylcholine neurotransmitter release. Typical clinical symptoms of all forms of botulism include cranial muscle paralysis, such as difficulty in speech, double vision and dilated pupils, desiccated mouth, difficulty in swallowing and speaking, and facial paralysis. Paralysis of the limbs and respiratory dysfunction becomes apparent when the disease progresses. Respiratory muscle paralysis can ultimately lead to death. Recuperation occurs upon the sprouting of transitory nerve endings that are located in when the synaptic activity of the original nerve regenerates. Recovery time may be numerous weeks to months and is dependent on the quantity of toxin ingested and to a lesser extent on the toxin type in question. Type A toxin tends to be more lethal than types B and $\mathrm{E}$ and causes the longest-lasting disease [18].

Food-Borne Botulism: The traditional form of botulism is food poisoning, an intoxication that follows the utilization of food containing preformed BoNT. Depending on the toxic dose, the incubation period may diverge from 12 to $72 \mathrm{~h}$. In addition to the common signs of

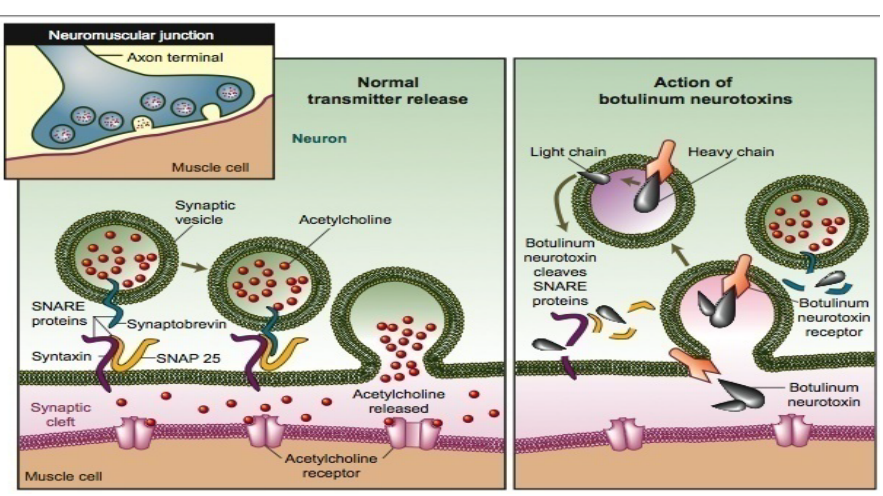

Figure 7. In normal condition - exocytosis process of acetylcholine containing vesicles take place in neuromuscular junction results muscle contraction but in the case of intoxication - botulinum neurotoxin cleave SNARE proteins (SNAP-25, VAMP-2 and syntaxin) which block exocytosis process of acetylcholine into synaptic cleft as a result muscle is going to paralyze 
botulism, the food-borne form may be identified by gastrointestinal symptoms such as vomiting, nausea and constipation. The treatment contains mainly of intensive symptomatic concern, including respiratory support. Intravenous administration of precise trivalent antitoxin towards $\mathrm{A}, \mathrm{B}$ and $\mathrm{E}$ toxins may be used to neutralize the circulating toxin. Nevertheless, the success of the antitoxin treatment is strongly dependent on the time of administration; if the toxin has entered the nerve endings and vanished from blood circulation, the treatment will be irrelevant. Furthermore, due to the threat of brutal allergic reactions to the antiserum, it is no longer used in some countries. Typical differential diagnoses comprise Guillain-Barré syndrome, MillerFisher syndrome, chemical intoxication, stroke and staphylococcal food poisoning. Assumed drug and alcohol abuse may infrequently prolong the time for a diagnosis to be made. Compared with foodborne sickness or death due to more widespread pathogens, such as Campylobacter, Salmonella, and Clostridium perfringens, food-borne botulism outbreaks are comparatively rare. On the other hand, regions with a high occurrence of botulism that causes a significant public health hazard include the Georgia, Poland, Russia, China, Kyrgyzstan, and certain ethnic populations in the North, e.g., Alaskan Inuits., Italy, Japan, Portugal, Germany, France and the former Yugoslavia have also reported numerous cases. The foods most recurrently involved, if traced, are home-made items such as cured meats, fermented fish products and canned vegetables. These outbreaks are typically sporadic and restricted to a family. Marketable foods have less frequently been implicated in food-borne botulism but such outbreaks may be big and they cause important economic losses to the food industry.Food-borne botulism case-fatality rate in developed countries is 5 to $10 \%$. Universal, more than half of the cases (52\%) were due to type B toxin followed by types A and E accounting for 34\% and 12\% respectively, in 1995. On exceptional occasions, type $\mathrm{F}$ toxin has been linked with food-borne botulism.

Infant Botulism: Unlike food-borne botulism, the additional types of human botulism are actually infections where the toxigenesis occurs in vivo. Infectious botulism is associated mainly to group I $C$. botulinum, as its temperature optimum for growth and subsequent toxin construction is close to the body temperature, whilst the growth of group II organisms at the same temperature is restricted. For infant botulism, in addition to group I C. botulinum types A, B, Bf, and F, cases due to type $\mathrm{E}$ and $\mathrm{F}$ toxins formed by Clostridium butyricum and Clostridium baratii respectively, have been reported. Infant botulism naturally affects babies under 1 year of age, with the youngest reported patient being only $54 \mathrm{~h}$ old. The circumstance develops as a consequence of ingesting spores of toxin-producing clostridia. As the gut micro-flora of small babies is weakly developed, C. botulinum spores may germinate and form a toxin in the large intestine. The clinical signs of infant botulism vary from a subclinical state to sudden death. It frequently begins with constipation that can last for many days, followed by the distinctive flaccid paralysis manifested by poorly feeding due to muscle weakness in the mouth and throat, ptosis, facial muscle paralysis and general weakness. Infant botulism has been recommended to be a causative agent of crib death. The major treatment for infant botulism is high-quality supportive care, with special focused on the patient's nutrition and respiratory functions. The use of antitoxin is frequently not required and the case-fatality rate is less than $2 \%$. Nevertheless, since 2003 a human-derived immune globulin (Baby BIG) has been accessible in the United States and has been revealed to significantly cut down the hospitalization period and reduce the treatment costs. The only foodstuffs that have been connected with infant botulism are honey which may hold high numbers of C. botulinum spores and infant milk powder. Medicinal plants, Dust and other materials in the environment also seem to be important sources of spores [19].

Wound Botulism: Wound botulism is an infrequent form of botulism even though it is increasingly diagnosed among injecting drug abusers due to the employ of contaminated needles or impure heroin. Wound botulism follows when C. botulinum spores germinate and multiplying in deep wounds or abscesses that provide anaerobic conditions. The clinical result is similar to the food-borne form, albeit with a lack of gastrointestinal signs. Wound botulism is frequently accompanied by a mixed infection and may be due to more than one BoNT producing strain. The median incubation period is 7 days. Moreover to respiratory support the treatment of wound botulism includes thorough antibiotics, surgical debridement and the administration of antitoxin. The predictable case-fatality rate is $15 \%$ [20].

Infectious Botulism in Adults: The adult form of transferable botulism is uncommon and resembles infant botulism in its pathogenesis and clinical status. It is a consequence of colonization of gastro - intestinal tract by toxin-producing clostridia. People with distorted intestinal flora due to abdominal surgery, long time antimicrobial treatment or gastrointestinal wounds and abscesses are particularly susceptible. Infectious botulism in adults is differentiated from the food-borne form by a dissimilar history of food consumption, with a lacking association to foods with high risk of botulism [21].

Other Forms of Botulism: Inhalation botulism may consequence of aerosolization of the neurotoxins. A few human cases have been reported. Iatrogenic botulism generalized weakness or local is rare but can expand as a consequence of therapeutic injection of the neurotoxin. A distinctive "outbreak" of iatrogenic botulism was caused by maladroit botulinum toxin treatment caused botulism in four people in November 2004 in Florida.

\section{Treatment}

Outcome is based on near the beginning diagnosis and treatment. Supportive care (including mechanical ventilation, airway protection and feeding by central tube or parenteral nutrition) and judicious administration of equine botulinum antitoxin are keys to the victorious management of botulism. Establish a means of communication early because occasionally conditions such as unbearable headaches are not communicated after the onset of paralysis.

\section{Vaccine research for botulism}

BoNT is an amazingly powerful substance that is responsible for the disease botulism. Although botulism is a comparatively rare disease in humans and animals, the mortality rate is increased without instant and proper treatment. Because of its intense potency and lethality, BoNT can also be employed as a biological weapon; such weapons are utilized by bioterrorists to create severe civic disruption, social anxiety and economic loss. Therefore, there is a need for developing vaccines against botulism.

BoNT toxoid vaccines: The achievement of formalized diphtheria and tetanus toxin as a means to build up a toxoid-based vaccine that protected from diphtheria and tetanus respectively provoked research into the feasibility of forming a vaccine against botulism utilizing the analogous approach. Initial attempts to manufacture a vaccine against botulism utilized formalin-treated crude C. botulinum filtrate. Formalin treated Liquid filtrates retained immunogenicity and eliminated toxicity. Animals that received frequent doses of formalin-treated 
neurotoxin were resistant to deadly challenge by the homologous BoNT serotypes. An extra step of toxin purification used alum precipitation that improved toxoid purity, whilst retaining the capacity to elicit a defensive immune response in animals. Frequent immunization with formalized, alum-precipitated toxoid established immunity. Even though local and systemic response to the initial vaccination with this BoNT toxoid was comparatively mild, the frequency and intensity of systemic reaction improved in individuals who received a second inoculum which stimulated the development of numerous approaches to lessen reactivity. One procedure that used sodium chloride, acid and cold ethanol precipitation of crude culture filtrate resulted in a pure, crystalline form of BoNT toxoid that enhanced the measurable neutralizing immune response in animals. A pentavalent formulation of BoNT (A, B, C, D and E) was useful against the homologous serotypes with least reactivity following immunization nevertheless; the supply of this vaccine is limited. Advances in molecular biological approaches give tools to divide the molecular mechanism of BoNT neutralization and need to develop a new generation of BoNT subunit vaccines with improved efficacy and safety [22].

Recombinant vaccines against BoNT: Helting and Nau in 1984, demonstrated that protein fragments formed from papain-digested tetanus toxin (TeNT) elicited defensive immunity in mice. A few years later, Fair weather and coworkers proved that partially purified recombinant fragment $\mathrm{C}$ from tetanus toxin $(\mathrm{rTeNT}(\mathrm{HC})$ ) protected mice when challenged with 10 LD50 of tetanus toxin. Since high sequence and structural homology that exists between the clostridial neurotoxins secreted by $C$. tetani and $C$. botulinum, applied this approach to developing a vaccine to protect against botulism. Then researchers sub cloned segments of the BoNT/A gene and express these harmless fragments in Escherichia coli followed vaccinate mice with the non-hazardous recombinants and test their capability to elicit protective immunity in vivo. Clones pCBA2, pCBA3, and pCBA4 (gift from Nigel Minton) comprising overlapping gene fragments of the BoNT/A gene, were the source for templates used to create the variety of antigens to be examined. Lesser versions of the gene fragments were made by cutting with restriction enzymes. Specific gene segments were formed by PCR. Expressed products were identified by SDS-PAGE and immunoblotting analyses, partly purified and used to vaccinate mice. Antigens representing a variety of regions from the three functional domains (binding and translocation domain of heavy chain at the $\mathrm{C}$ and $\mathrm{N}$ terminus respectively and catalytic domain of light chain) of the toxin were analyzed for their capability to elicit protective antibodies in mice. All the fragments tested, out of three only one was able to entirely protect mice. This was the fragment positioned at the carboxy-terminal of the BoNT ( $50 \mathrm{kDa})$ designated as the fragment $\mathrm{C}$ region. Our succeeding efforts to develop vaccine candidates to defend against BoNT forthwith focused totally on the HC region. One could imagine the most perfect recombinant BoNT vaccine candidate would be the smallest, non-hazardous fragment of the toxin able of eliciting noteworthy protective immunity, while being totally devoid of any noticeable biological property connected with its parent neurotoxin (receptor binding, translocation and enendoproteolytic activity). One strategy to a recombinant BoNT vaccine has been planned which would utilize a mutated holotoxin as the immunogen. In this strategy, the endopeptidase activity of the light chain would be removed by changing the amino acid residues necessary for zinc binding (H229 $\rightarrow \mathrm{G}$ and $\mathrm{H} 233 \rightarrow \mathrm{N})$ and the $\mathrm{H}_{2} \mathrm{O}$ binding $(\mathrm{E} 230 \rightarrow \mathrm{T})$. The resultant immunogen however, would still hold binding and entrance activities. Researches believed the withholding of those activities could be challenging in terms of their effects on protection and the extra testing we might be requisite to do to persuade the Center for Biologics Evaluation and Review (CBER) at FDA that this manufactured goods was safe. And indeed, at a pre-IND meeting on April, 1999, held to appraisal the recombinant BoNT (HC) vaccines, a CBER board voiced concern as to the potential of the assumed $\mathrm{HC}$ vaccine to cause adverse neurological disorders and reactions due to its binding to specific ligand on cholinergic nerve cells. Even though in competition studies, BoNT/A and BoNT/ B (HC) vaccines did not fight with the neurotoxin for receptor binding (unpublished data), the CBER panel suggested we do extra testing (e.g., Functional Observational Battery beyond the acute toxicology testing. So need to generate new generation vaccines against botulism $[23,24]$.

DNA vaccine: A DNA vaccine was made which expressed the binding domain (HC) of C. botulinum neurotoxin serotype F merged to a signal peptide. Intra-muscular 3-doses fully protected $B A L B / c$ mice in opposition to $10^{(4)} \mathrm{MLD}$ of serotype $\mathrm{F}$ toxin. DNA vaccination priming immune response followed by a single booster with type $\mathrm{F}$ binding domain protein generates high levels of antibody against the neurotoxin binding domain. This study showed the efficacy of DNA vaccination for protection against BoNT/ F and reveal that a prime-boost regimen could be a well-organized method of generating antibody for passive immune therapy in cases of BoNT/F toxin involving botulism. Human botulism is commonly associated with serotypes A, B, E and F. This suggests that the maximum need is for a tetravalent vaccine that provides defense against all four of these serotypes. Researcher investigated the feasibility of generating numerous tetravalent vaccines that protected mice against the four serotypes. Initially, monovalent replicon vaccine against BoNT created better antibody response and protection than that of corresponding conservative DNA vaccine. Secondly, dualexpression DNA replicon (replicon particle VRP-E/FHc vaccine or pSCARSE/FHc) was well resistant to the challenge of botulinum neurotoxin $\mathrm{E}$ and $\mathrm{F}$ mixture as a combination vaccine contained of two monovalent replicon vaccines. In conclusion, the dual-expression DNA replicon or replicon particle tetravalent vaccine could concurrently and successfully neutralize and protect the four BoNT serotypes. Protection interrelated directly with serum ELISA titers and neutralization antibody levels to BoNTs. So, replicon-based DNA might be efficient vector to develop BoNT vaccines, which might be more attractive for use in clinical application than the conventional DNA vaccines. This study showed the utility of combining dual-expression DNA replicon or replicon particle vaccines into multi-agent formulations as effective tetravalent vaccines for forming protective responses to four serotypes of BoNTs [25-27,29,30].

\section{References}

1. Barash JR, Arnon SS (2004) Dual toxin-producing strain of Clostridium botulinum type Bf isolated from a California patient with infant botulism. J Clin Microbiol 42: 1713-1715. [Crossref]

2. Sobel J (2005) Botulism. Clin Infect Dis 41: 1167-1173. [Crossref]

3. Barash JR, Arnon SS (2014) A novel strain of Clostridium botulinum that produces type $\mathrm{B}$ and type H botulinum toxins. J Infect Dis 209: 183-191. [Crossref]

4. Sebaihia M, Peck MW, Minton NP, Thomson NR, Holden MTG, et al. (2007) Genome sequence of a proteolytic (Group I) Clostridium botulinum strain Hall A and comparative analysis of the clostridial genomes. Genome Res 17: 1082-1092. [Crossref]

5. Ting PT, Freiman A (2004) The story of Clostridium botulinum: from food poisoning to Botox. Clin Med (Lond) 4: 258-261. [Crossref]

6. Simidu U (2001) An introduction to predictive microbiology. Shokuhin Eiseigaku Zasshi 42: J317-323.

7. Chaudhry R, Dhawan B, Kumar D, Bhatia R, Gandhi JC, et al. (1998) Outbreak of suspected Clostridium butyricum botulism in India. Emerg Infect Dis 4: 506-507. [Crossref] 
8. Agarwal AK, Goel A, Kohli A, Rohtagi A, Kumar R (2004) Food-borne botulism. $J$ Assoc Physicians India 52: 677-678. [Crossref]

9. Lalitha KV, Surendran PK (2002) Occurrence of Clostridium botulinum in fresh and cured fish in retail trade in Cochin (India). Int J Food Microbiol 72: 169-174. [Crossref]

10. Sathish S, Swaminathan K (2009) Genetic diversity among toxigenic clostridia isolated from soil, water, meat and associated polluted sites in South India. Indian J Med Microbiol 27: 311-320. [Crossref]

11. Dhaked RK, Sharma SK, Parida MM, Singh L (2002) Isolation and characterization of Clostridium botulinum type E from soil of Gwalior, India. J Nat Toxins 11: 49-56. [Crossref]

12. Halliwell J, Gwenin C (2013) A label free colorimetric assay for the detection of active botulinum neurotoxin type A by SNAP-25 conjugated colloidal gold. Toxins (Basel) 5: 1381-1391. [Crossref]

13. Arnon SS, Schechter R, Inglesby TV, Henderson DA, Bartlett JG, et al. (2001) Botulinum toxin as a biological weapon: medical and public health management. JAMA 285: 1059-1070. [Crossref]

14. Rossow H, Kinnunen PM, Nikkari S (2012) Botulinum toxin as a biological weapon Duodecim 128: 1678-1684. [Crossref]

15. Fujinaga Y (2010) Interaction of botulinum toxin with the epithelial barrier. J Biomed Biotechnol 2010: 974943. [Crossref]

16. Elias M, al-Saleem F, Ancharski DM, Singh A, Nasser Z, et al. (2011) Evidence that botulinum toxin receptors on epithelial cells and neuronal cells are not identical: implications for development of a non-neurotropic vaccine. J Pharmacol Exp Ther 336: 605-612. [Crossref]

17. Yao G, Lee K, Gu S, Lam KH, Jin R (2014) Botulinum neurotoxin A complex recognizes host carbohydrates through its hemagglutinin component. Toxins (Basel) 6: 624-635. [Crossref]

18. Lindstrom M, Korkeala H (2006) Laboratory diagnostics of botulism. Clin Microbiol Rev 19: 298-314. [Crossref]

19. Rosow LK, Strober JB (2015) Infant botulism: review and clinical update. Pediatr Neurol 52: 487-492. [Crossref]
20. Cherington M (2004) Botulism: update and review. Semin Neurol 24: 155-163. [Crossref]

21. Sheppard YD, Middleton D, Whitfield Y, Tyndel F, Haider S, et al. (2012) Intestinal toxemia botulism in 3 adults, Ontario, Canada, 2006-2008. Emerg Infect Dis 18: 1-6. [Crossref]

22. Vlata Z, Tsatsakis A, Tzagournissakis M, Krambovitis E (2012) Evaluation of specific immune responses to BoNT/A and tetanus toxoid in patients undergoing treatment for neurologic disorders. Endocr Metab Immune Disord Drug Targets 12: 268-273. [Crossref]

23. Smith LA (1998) Development of recombinant vaccines for botulinum neurotoxin Toxicon 36: 1539-1548. [Crossref]

24. Webb RP, Smith TJ, Wright PM, Montgomery VA, Meagher MM, et al. (2007) Protection with recombinant Clostridium botulinum $\mathrm{C} 1$ and $\mathrm{D}$ binding domain subunit (Hc) vaccines against $C$ and D neurotoxins. Vaccine 25: 4273-4282. [Crossref]

25. Yu YZ, Zhang SM, Sun ZW, Wang S, Yu WY (2007) Enhanced immune responses using plasmid DNA replicon vaccine encoding the Hc domain of Clostridium botulinum neurotoxin serotype A. Vaccine 25: 8843-8850. [Crossref]

26. Li N, Yu YZ, Yu WY, Sun ZW (2011) Enhancement of the immunogenicity of DNA replicon vaccine of Clostridium botulinum neurotoxin serotype A by GM-CSF gene adjuvant. Immunopharmacol Immunotoxicol 33: 211-219. [Crossref]

27. Tian DY, Sun Y, Wai SF, Lee FK, Qi-Lin M, et al. (2012) Enhancement of the immunogenicity of an alphavirus replicon-based DNA vaccine against classical swine fever by electroporation and coinjection with a plasmid expressing porcine interleukin 2. Vaccine 30: 3587-3594. [Crossref]

28. Frevert J, Dressler D (2010) Complexing proteins in botulinum toxin type A drugs: help or a hindrance? Biologics 4: 325-332. [Crossref]

29. Mukherjee J, Dmitriev I, Debatis M, Tremblay JM, Beamer G, et al. (2014) Prolonged prophylactic protection from botulism with a single adenovirus treatment promoting serum expression of a VHH-based antitoxin protein. PLoS One 9: e106422. [Crossref]

30. Vanella de Cuetos EE, Fernandez RA, Bianco MI, Sartori OJ, Piovano ML, et al. (2011) Equine botulinum antitoxin for the treatment of infant botulism. Clin Vaccine Immunol 18: 1845-1849. [Crossref]

Copyright: (C2021 Sharma A. This is an open-access article distributed under the terms of the Creative Commons Attribution License, which permits unrestricted use, distribution, and reproduction in any medium, provided the original author and source are credited. 